ORIGINAL ARTICLE

\title{
Investigating the cytotoxicity of four types of freshly prepared root canal sealers using human gingival fibroblasts
}

\author{
EHSAN MOSTAJERAN, SHIRIN ASGARANI, HAMID RAZAVIAN, ABBASALI KHADEMI \\ ${ }^{1}$ Resident of Oral and Maxillofacial Surgery, Ahvaz Jundishapur University of Medical Sciences, Ahvaz, Iran \\ ${ }^{2}$ Pediatric Dentist, Ahvaz Jundishapur University of Medical Sciences, Ahvaz, Iran. \\ ${ }^{3}$ Dental materials Research Center and Departments of Endodontics, School of Dentistry, Isfahan University of Medical Sciences, Isfahan, \\ Iran. \\ ${ }^{4}$ Torabinejad Research Center, Department of Endodontics, School of Dentistry, Isfahan University of Medical Sciences, Isfahan, Iran
}

\begin{abstract}
Introduction: One of the most important characteristics of endodontic treatment seals is their biocompatibility. The aim of present study was to investigate the cytotoxicity of four types of freshly prepared root canal sealers using human gingival fibroblasts.

Materials and Methods: In this experimental laboratory study, freshly prepared sealers were investigated. To investigate the cytotoxicity, each sample was extracted separately in RPMI-1640 (Sigma Aldrich-USA). Then, the resulting extracts were exposed to $\mathrm{C} 165$ fibroblast cells in culture medium for 1, 3 and 7 days. Then, the level of cytotoxicity was assessed by MTT colorimetric method based on the level of light absorption. In the present study, multivariate analysis of variance was used to analyze the data.

Results: The results showed that the cytotoxicity of $\mathrm{AH} 26$ and tgsealer sealers decreased and MTA Fillapex sealer increased over time. Also, Adseal sealer did not follow a logical trend but, in general, its cytotoxicity increased over time. In addition, based on mean of all data obtained from each sealer, MTA Fillapex had the lowest cytotoxicity and Adseal had the highest cytotoxicity, and $\mathrm{AH} 26$ cytotoxicity was higher than that of tgsealer.

Conclusion: According to the results of the present study, the MTA-based sealer of MTA Fillapex has the lowest cytotoxicity.

Keywords: Root canal sealers, fibroblasts, freshly prepared roots
\end{abstract}

\section{INTRODUCTION}

The materials commonly used to fill the root canal can be divided into solid phase (gutta-percha) and cementing materials (sealer). The presence of a sealer, as a core material, is essential in all methods, regardless of the presence of a central material. It provides a strong seal against fluids (1). Grossman describes the characteristics of an ideal sealer. At current, there is no sealer to have all of these characteristics (1). According to Grossman, one of the characteristics of a suitable sealer is the appropriate biocompatibility of the sealer. According to him, sealer should have good biocompatibility to prevent secondary problems after root canal treatment (2). Textile compatibility is one of the most important characteristics of canal fillers, because the release of certain components of sealer ingredients may cause various reactions in the periapical tissue $(3,4,5,6,7,8)$. Sealers can be in close contact with living tissues due to their viscosity behavior. Therefore, the biological properties of these materials are important. A material that does not have good biocompatibility has the potential for cytotoxicity, and materials with cytotoxicity can cause inflammatory responses and tissue damage [9]. However, the sealer should be well tolerated by periapical tissue, not delay tissue repair, and stimulate repair of damaged structures. Cytotoxicity can be determined by MTT colorimetric test. This test detects only living cells that make it possible to measure cytotoxicity, proliferation, or cellular activity (11).

In a study conducted by Eldeniz et al. (2007) to compare the cytotoxicity levels of five new sealers (RC Sealer, Epiphany, EndoREZ, GuttaFlow, Acroseal) and conventional sealers (AH Plus, RoekoSeal and Apexit), the cytotoxicity levels of the sealers on human gingival fibroblasts (HGF's) and mouse fibroblasts (L929) by using MTT diagnostic test were determined. Accordingly, AHPlus sealer had no toxic effect in any of the cases, and even in hardened samples, it stimulated cell proliferation. Resinbased (Endorez, Epiphany) and calcium hydroxide-based sealers (Apexit, Acroseal) had the highest cytotoxicity on L929 and HGF, but the level of cytotoxicity by L929 cells on Apexit and Asexit sealers had higher sensitivity and ReakoSeal and Gutta Flow had moderate cytotoxicity (12). In a study conducted by Claudia et al. (2012), the level of cytotoxicity of MTA FillApex and AHPlus sealers with white MTA on Chinese hamster fibroblasts was assessed by MTT diagnostic test. AHPlus and MTA FilApex sealers at concentrations of 1 : 1 and 1: 2 had a high cytotoxicity during 72,48 and 12 hours and with decreasing concentration to $1: 4$ to $1: 32$, the toxicity decreased. However, in general, the level of cytotoxicity of FillApex MTA was higher than that of $\mathrm{AH}$ plus at similar concentrations and times. White MTA also had low cytotoxicity at different concentrations and times (13). In a recent study conducted by Yoshino et al. (2013), they assessed the cytotoxicity of Milla Fillapex sealer with White MTA and Portland cement on periodontal fibroblast cells. The cytotoxicity of White MTA was moderate and that of Portland cement was low, but cytotoxicity of MTA Fillapex was high (14). Given what was stated above, the aim of this study was to investigate the cytotoxicity of four types of freshly prepared root canal sealers using human gingival fibroblasts.

\section{MATERIALS AND METHODS}

This experimental-laboratory study was conducted in Isfahan University of Medical Sciences, Faculty of 
Dentistry, Professor Torabinejad Dental Science Research Center and Histology Laboratory of Isfahan University of Medical Sciences. In the present study, AH 26 (DENTSPLY DeTrey, GmbH, Konstanz, Germany), tgsealer (Technical \& General Ltd., London, United Kingdom), MTA Fillapex (Angelus IPO, Londrina, Brazil), Adseal (Meta biomed, Chungbuk, Republic of Korea) sealers were used. In the present study, multivariate analysis of variance was used to analyze the data. To examine the data obtained from the MTT test, the samples were first divided into 10 groups.

Group 1: includes samples of freshly prepared $\mathrm{AH} 26$ sealer

Group 2: includes samples of freshly prepared MTA Fillapex sealer

Group 3: includes samples of freshly prepared Adseal sealer

Group 4: includes samples of freshly prepared tgsealer sealer

Group 5: Includes negative control samples (containing gingival fibroblast cells and RPMI culture medium without adding any other material)

In each group, 9 data were obtained, 3 data were related to incubation in 1 day (which its light absorption was measured 24 hours after adding the sealer extract to the cells) and also 3 data were related to the sample of 3-day samples and 3 data were related to 7-day samples.

\section{Method of cell culture and passage}

Living cells were cultured in small $25 \mathrm{~T}$ flasks. Cells were cultured in an incubator and the culture medium was replaced every three days under a laboratory hood (Jahl Iran) to cover the bottom of the flask in a monolayer manner. When the cells reached 70 to $80 \%$ density, they were trypsinized with $2 \mathrm{ml}$ of EDTA-Try (Ethylene Diamine Tetraacetic Acid-Trypsin) for 2 to 5 minutes at $37^{\circ} \mathrm{C}$ to separate the cells from the bottom of the flask. Then, RPMI-1640 medium was immediately added to the flask at least at its volume to neutralize the enzyme, and was centrifuged at $1800 \mathrm{rpm}$ for 5 minutes. Then, the medium was removed from the precipitated cell sediments and the medium culture was poured again on the cell sediments and a cell suspension was made in the tube with the Vertex apparatus (VEIP-zx3 Italy) and the contents of the tube were poured into two new flasks as the next passage cells and placed in the incubator. The flasks were checked every day and it lasted almost 4 to 5 days to become monolayer

\section{Melting}

First, the freeze vial was removed from the nitrogen tank. Then, it was placed in distilled water at $37^{\circ} \mathrm{C}$, and was cultured in $10 \mathrm{ml}$ of RPMl 1640 culture medium with cell suspension serum and was placed in a $37^{\circ}$ incubator at $5 \%$ carbon dioxide pressure. After 24 hours, the medium was replaced and placed again in the incubator until the cells were grown fully and became ready for trypsinization.

\section{Trypsinization}

When $50-60 \%$ of the flask was full of grown cells, the cells were separated from the bottom of the flask using Try / EDTA enzyme, and were poured into a Falcon tube and were centrifuged. The supernatant was drained and the cells were placed in $2 \mathrm{ml}$ of suspension and the cells were counted using a neobar slide.

\section{Sealer preparation steps}

The endodontic freshly prepared sealers used in this study were tested. For preparation, the sealers were mixed under aseptic conditions based on the manufacturer's instructions. Then, the mixed sealers were poured into sterile Teflon generators with a diameter of $7 \mathrm{~mm}$ and a height of $3 \mathrm{~mm}$. The tablet of newly mixed sealers was completely removed from Teflon generators. The sealers prepared in RPMI-1640 were immersed at a ratio of 1.25 $\mathrm{cm} 2$ / $\mathrm{ml}$ (surface of sealer samples to volume of RPMI) for 24 hours at $37{ }^{\circ} \mathrm{C}$ for extraction. Then, the extract was filtered through a $0.2 \mu \mathrm{m}$ sterile filter (Biotech, Germany) and the extract was prepared for proximity to the cells. The cells were poured into 12-cell culture media with 20000 cells per cell. Then, the plates were incubated for 24 hours. Then, the medium was emptied and $1 \mathrm{ml}$ of the extracted medium was added to each cell, and after 1, 3, and 7 days, cell survival in culture medium was measured (Figure 1). To control the conditions for all three times, the medium was cultured with pure medium.

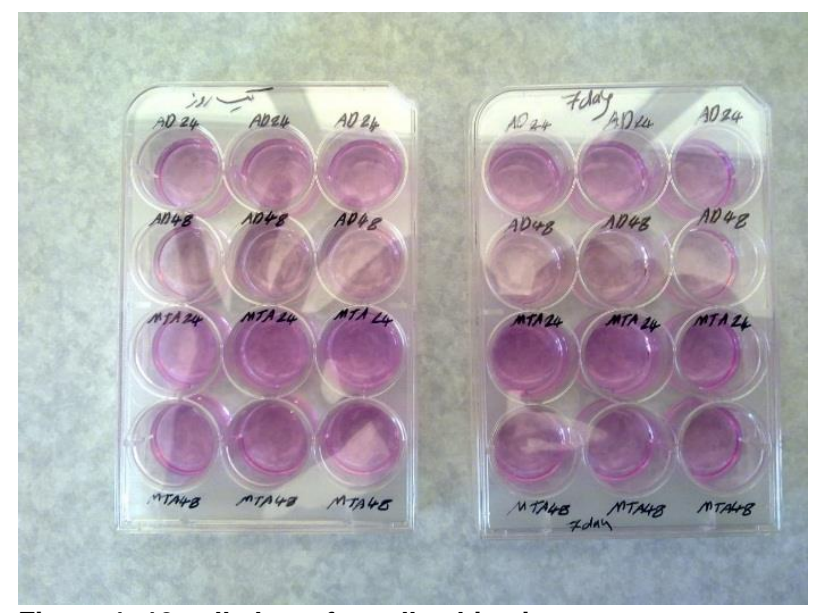

Figure 1: 12-cell plates for cell cultivation

\section{MTT test}

The results of this test determine the number of living cells and level of metabolic activity of the cells. Thus, the MTT method is a sensitive index for assessing the cytotoxicity of dental materials (15). This test is one of the most common methods for examining the cytotoxicity of drugs, oral disinfectants and peroxide-containing products. The advantage of this method is its simplicity, speed and repeatability (16). After 1,3 and 7 days, the medium was drained and washed with PBS (Phosphate-Buffered Saline). Then, $400 \mu \mathrm{l}$ of medium and $40 \mu \mathrm{l}$ of MTT were added and kept in the incubator for 24 hours. Then, the medium was gently drained and $400 \mu$ of DMSO (Dimethyl Sulfixide) was added to it and was kept at darkness for 2 hours. Then, the solution was transferred to a 96-cell plate and the quantity of the obtained formazan was determined by spectrophotometry at a wavelength of $570 \mathrm{~nm}$. After measuring the level of light absorption in each culture medium, the survival rate of cells was reported as a percentage compared to the control group in which all cells survived. 


\section{RESULTS}

The results of the photometry test showed the metabolic activity of the cells. Table 1 presents the level of light absorption of different groups.

Table 1: mean light absorption of samples

\begin{tabular}{|c|c|c|c|}
\hline day & Sealer & $\begin{array}{l}\text { Freshly } \\
\text { sealers }\end{array}$ & prepared \\
\hline \multirow[t]{5}{*}{1} & $\mathrm{AH} 26$ & 0.239 & \\
\hline & MTA Fillapex & 0.598 & \\
\hline & Adseal & 0.536 & \\
\hline & tgsealer & 0.290 & \\
\hline & Negative control & 0.627 & \\
\hline \multirow[t]{5}{*}{3} & $\mathrm{AH} 26$ & 0.363 & \\
\hline & MTA Fillapex & 0.596 & \\
\hline & Adseal & 0.363 & \\
\hline & tgsealer & 0.563 & \\
\hline & Negative control & 0.779 & \\
\hline \multirow[t]{5}{*}{7} & $\mathrm{AH} 26$ & 0.413 & \\
\hline & MTA Fillapex & 0.434 & \\
\hline & Adseal & 0.398 & \\
\hline & tgsealer & 0.466 & \\
\hline & Negative control & 0.557 & \\
\hline
\end{tabular}

The level light absorption in the positive control group was zero in all samples. To report the cytotoxicity of sealers in the mentioned groups, the mean light absorption of each sample in each group was divided by the level of optical absorption of the negative control group of the same group. Table 2 presents the cytotoxicity of these groups (2).

Table 2: Freshly prepared samples

\begin{tabular}{|l|l|l|l|}
\hline day & sealer & SD \pm mean & Cytotoxicity \\
\hline \multirow{4}{*}{1} & AH 26 & $0.37 \pm 0.83$ & moderate \\
\cline { 2 - 4 } & MTA Fillapex & $0.95 \pm 0.7$ & no \\
\cline { 2 - 4 } & Adseal & $0.85 \pm 0.11$ & low \\
\cline { 2 - 4 } & tgsealer & $0.46 \pm 0.11$ & moderate \\
\hline \multirow{4}{*}{3} & AH 26 & $0.47 \pm 0.10$ & moderate \\
\cline { 2 - 4 } & MTA Fillapex & $0.76 \pm 0.7$ & low \\
\cline { 2 - 4 } & Adseal & $0.47 \pm 0.8$ & moderate \\
\cline { 2 - 4 } & tgsealer & $0.72 \pm 0.15$ & low \\
\hline \multirow{4}{*}{7} & AH 26 & $0.74 \pm 0.23$ & low \\
\cline { 2 - 4 } & MTA Fillapex & $0.61 \pm 0.4$ & low \\
\cline { 2 - 4 } & Adseal & $0.51 \pm 0.1$ & moderate \\
\cline { 2 - 4 } & tgsealer & $0.83 \pm 0.11$ & low \\
\hline
\end{tabular}

The cytotoxicity of $\mathrm{AH} 26$ and tgsealer sealers decreased and cytotoxicity of MTA Fillapex sealer increased over time. Also, Adseal sealer did not follow a logical trend, but in general, its cytotoxicity increased over time.

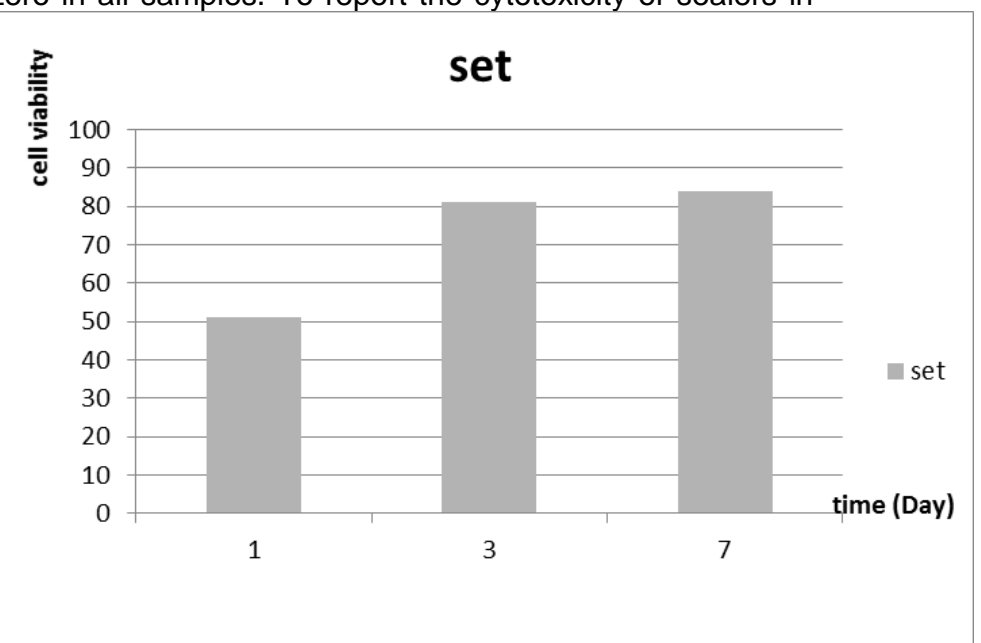

Chart 1: Samples of AH 26 


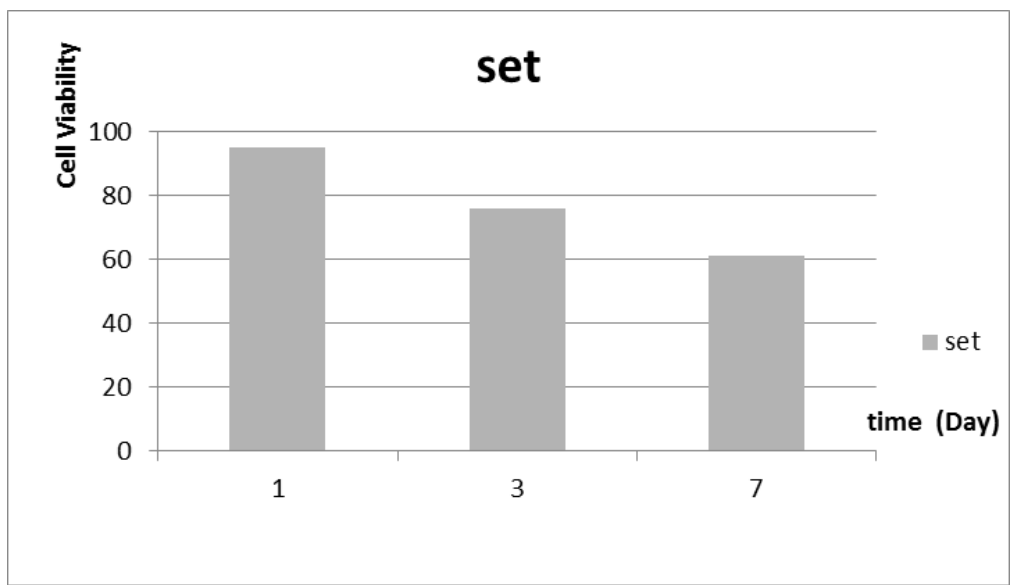

Chart 2: Samples of MTA Fillapex

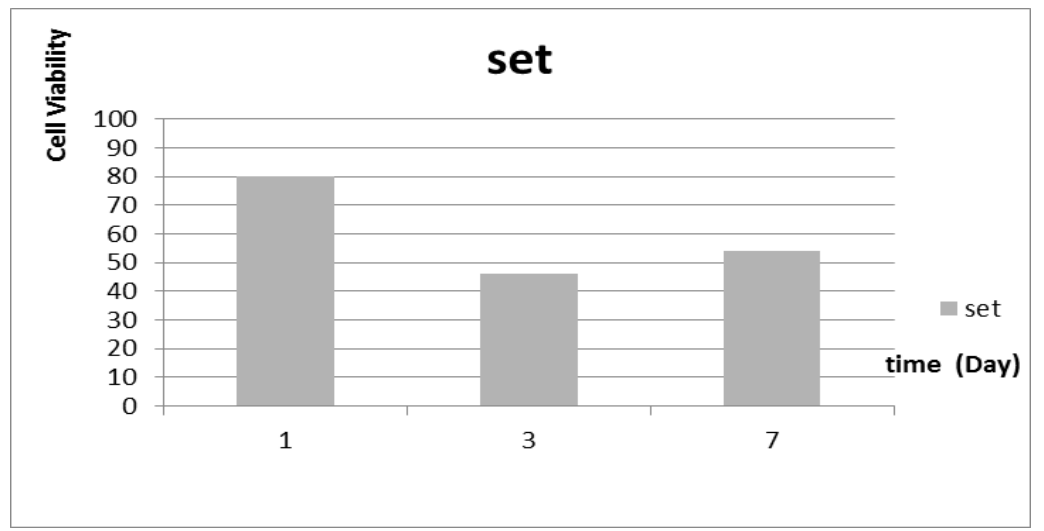

Chart 3: samples of Adseal

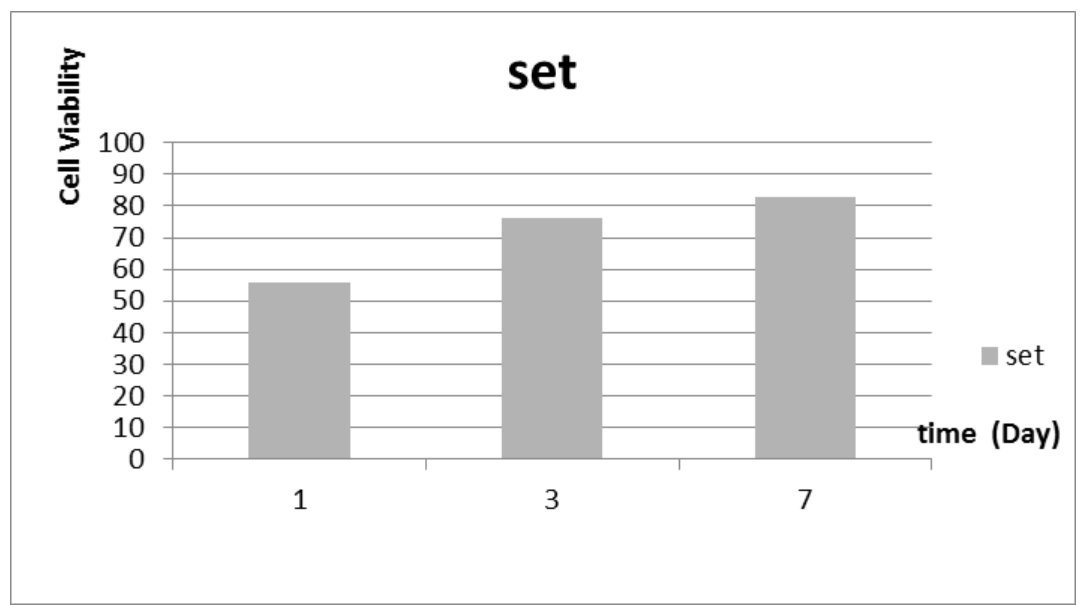

Chart 4: samples of tgsealer

According to the statistical test of multivariate analysis of variance, on average, all data obtained from each sealer, MTA Fillapex had the lowest cytotoxicity and Adseal sealer the highest cytotoxicity, and the cytotoxicity of $\mathrm{AH} 26$ was higher than that of tgsealer.

\section{DISCUSSION}

The aim of the present study was to investigate the cytotoxicity of four types of freshly prepared root canal sealers using human gingival fibroblasts. Clinically, freshly prepared sealers or the sealers that are in the incomplete polymerization stage enter the mouth. However, even after hardening, it is possible that potentially toxic compounds of these materials to be released after contact with tissue fluids (10). Thus, in various studies, it has been recommended to examine both hardened and freshly prepared sealers. The material from which the sealers are extracted for cell culture tests is effective in the cytotoxicity 
of the samples. In a study conducted by Schweikl et al., when AHPlus was exposed to $0.9 \%$ sodium chloride for extraction, it showed the lowest cytotoxicity and when exposed to DMSO, it showed the highest cytotoxicity [17]. For this purpose, to eliminate the effect of DMSO, it is recommended sealers to be extracted in RPMI, which is the medium of cell culture, as it was used in the present study. In the present study, MTT method was used to assess the level of cytotoxicity. In the present study, the cytotoxicity of four sealers of $\mathrm{AH} 26$, Adseal, tgsealer and MTA Fillapex was tested in vitro on human gingival fibroblast cells in cell culture medium. The highest cytotoxicity was related to $\mathrm{AH} 26$ sealer on the first day and Adseal sealer on the third and seventh days, both of which are resin-based sealers. The lowest cytotoxicity was related to MTA Fillapex sealer. Also, in the study conducted by Yoshino et al., the cytotoxicity of White MTA was moderate and that of Portland Cement was low, but MTA Fillapex showed high cytotoxicity, which fibroblast cells of periodontal ligaments was also used in this study (14).

The results obtained on resin-based Adseal and $\mathrm{AH}$ 26 sealers in the present study showed that Adseal had the highest cytotoxicity, followed by $\mathrm{AH} 26$. In the present study, resin-based sealers had the highest cytotoxicity compared to other sealers. The AHPlus sealer is similar to the $\mathrm{AH} 26$, except that it does not contain formaldehyde. In different studies, different opinions have been reported about the AHPlus sealer. In the studies conducted by Eldeniz et al. and Karapinar et al., the level of cytotoxicity of AHPlus was very low this sealer even stimulated cell growth in some cases and times $(18,12)$. Tgsealer sealer another sealer examined in the present study. The cytotoxicity of this sealer was moderate and low and showed the lowest cytotoxicity after MTA Fillapex. Tgsealer sealer is a new ZEO-based sealer and due to its newness, there is little scientific information to compare it with other sealers, but other ZOE- based sealers can be used to compare and find out the properties of tgsealer.

\section{CONCLUSION}

Based on the results of the present study, MTA-based sealer of MTA Fillapex has the lowest cytotoxicity and Adseal resin-based sealer has the highest cytotoxicity, compared to other studied sealers.

\section{REFERENCES}

1. Cohen S, Hargreaves KM, Berman LH. Pathways of the pulp. 10 ed: Mosby; 2011. p. 260-65, 331, 358-62, 559-65, 582.
2. Ingle JI. Ingle's endodontics 6. Pm USA Ltd; 2008. p. 103040.

3. Bernath M, Szabo J. Tissue reaction initiated by different sealers. Int Endod J 2003;36:256-61.

4. Bouillaguet S, Wataha JC, Lockwood PE, Galgano C, Golay A,Krejci I. Cytotoxicity and sealing properties of four classes of endodontic sealers evaluated by succinic dehydrogenase activity and confocal laser scanning microscopy. Eur J Oral Sci 2004;112:182-7.

5. Harrison JW, Bellizzi R, Osetek EM. The clinical toxicity of endodontir medicaments. J Endod 1979;5:42-7.

6. Kaplan AE, Ormaechea MF, Picca M, Canzobre MC, Ubios AM.Rheological properties and biocompatibility of endodontic sealers. Int Endod J 2003;36:527-32.

7. Gençog^lu N, Turkmen C, Ahiskali R. A new silicon-based root canal sealer (Roekoseal-Automix). J Oral Rehabil 2003;30:753-7.

8. Hauman $\mathrm{CH}$, Love RM. Biocompatibility of dental materials used in contemporary endodontic therapy: a review. Part 2.Root-canal-filling materials. Int Endod J 2003;36:147-60.

9. Bertram JS. The molecular biology of cancer. Mol Aspects Med. 2000;21(6):167-223.

10. Huang FM, Tai KW, Chou MY, Chang YC. Cytotoxicity of resin-, zinc oxide-eugenol-, and calcium hydroxide-based root canal sealers on human periodontal ligament cells and permanent V79 cells. Int Endod J. 2002;35(2):153-8.

11. Meryon SD, Stephen PG, Browne RM. A modification of the Millipore method for screening restorative materials. Int Endodon J 1982; 15:197-202.

12. Eldeniz A, Mustafa K, Ørstavik D, Dahl J. Cytotoxicity of new resin-, calcium hydroxide-and silicone-based root canal sealers on fibroblasts derived from human gingiva and L929 cell lines. Int Endod J. 2007;40(5):329-37.

13. Bin CV, Valera MC, Camargo SEA, Rabelo SB, Silva GO, Balducci I, et al. Cytotoxicity and Genotoxicity of Root Canal Sealers Based on Mineral Trioxide Aggregate. J Endod. 2011;38(4):495-500.

14. Yoshino P, Nishiyama CK, Modena KCdS, Santos CF, Sipert CR. In Vitro Cytotoxicity of White MTA, MTA Fillapex® and Portland Cement on Human Periodontal Ligament Fibroblasts. Braz Dent J. 2013;24(2):111-6.

15. Mosmann T. Rapid colorimetric assay for cellular growth and survival: application to proliferation and cytotoxicity assays. J Immunol Methods 1983;65:55-63

16. Zhang et al. Evaluation of Cytotoxicity of MTAD Using the MTT-Tetrazolium Method. J of Endod 2003;29(10):654-657.

17. Schweikl H, Schmalz G. The induction of micronuclei in V79 cells by the root canal filling material $\mathrm{AH}$ plus. Biomaterials. 2000;21(9):939-44.

18. Karapınar-Kazandağ M, Bayrak Ö, Yalvaç M, Ersev H, Tanalp J, Şahin F, et al. Cytotoxicity of 5 endodontic sealers on L929 cell line and human dental pulp cells. Int Endod J. $2011 ; 44(7): 626-34 .$. 